\title{
On a magnetosphere disturbed by solar wind; observations of macroelectrons
}

\author{
E. B. Wodnicka \\ Space Reaserch Centre, PAS, Bartycka 18a, 00716 Warsaw, Poland
}

Received: 19 May 2008 - Revised: 27 March 2009 - Accepted: 6 May 2009 - Published: 8 June 2009

\begin{abstract}
Three-dimensional electromagnetic full kinetic particle code (a version of TRISTAN) is used to study the interaction of a weakly-magnetized object with a solar wind of low density. The details of two magnetospheric processes wave activity and energetic electrons appearing at the flanks of the magnetosphere - are presented. The results of the simulation are compared with known magnetospheric data.
\end{abstract}

Keywords. Magnetospheric physics (Magnetospheric configuration and dynamics; Planetary magnetospheres; Solar wind-magnetosphere interactions)

\section{Introduction}

Magnetospheres of magnetized objects of which we already know, result from the interaction of interplanetary plasma and electromagnetic fields with the plasma and fields existing in their vicinity. The most explored and understood is the magnetosphere of the Earth. Its large-scale MHD models are numerous (see e.g. Walker et al., 1993; Spicer et al., 1996; Slinker et al., 2001; Reader et al., 2001; De Zeeuw et al., 2001). Three-dimensional, electromagnetic, full kinetic particle-in-cell (PIC) global simulations of the magnetosphere were initiated by Buneman (1993) and are being continued by Nishikawa $(1997,2001)$ and Cai et al. (2006). The hermean magnetosphere was simulated by Kabin et al. (2000), Ip and Kopp (2002), Kallio and Janhunen (2003) and by Trávnicek et al. (2007). The similarities and differences between the two magnetospheres were studied by Delcourt et al. (2002).

Large-scale models of magnetized objects other than planets have been published recently. A 3-D multi-fluid representation of Ganymede's magnetosphere was published by

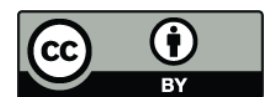

Correspondence to: E. B. Wodnicka (elwo@cbk.waw.pl)
Paty and Winglee (2004). Gallileo mission made accessible the space in the vicinity of asteroids (Kivelson et al., 1993; Wang and Kivelson, 1996; Blanco-Cano et al., 2003). A 2-D hybrid code was applied by Omidi et al. $(2002,2003)$, first to investigate asteroids, then to study dipolar magnetospheres in general. The authors demonstrated that the structure of the interaction region, described in terms of whistler and magnetosonic waves and plasma density distribution, depended on the dipole strength and on interplanetary magnetic field (IMF) orientation. Simon et al. (2006) simulated the plasma environment of a magnetized asteroid using a 3-D hybrid code, and elucidated kinetic details of the protons traversing the interaction region.

This study is a continuation of efforts to build up a PIC model for any magnetosphere. A weakly-magnetized celestial body, also referred to as an object, immersed in a fluctuating solar wind of low density is investigated. It is demonstrated that the response of its magnetosphere to the interplanetary medium is of a known nature; the events observed in the simulated system are typical for a magnetosphere. Two events are considered: waves excitation at the edge of the evening magnetopause, and the existence of energetic electrons at dawn. In the simulations the attributes of the wind vary in time and the object immersed in a quiet wind is disturbed in two different ways to distinguish number density disturbance from that induced by interplanetary fields. In both cases the bulk velocity of the solar wind is kept constant. In the former case, the number density increase results in a fluctuating current and waves of hydromagnetic nature are excited at the edge of the magnetosphere. In the latter case a short-lasting solar wind number density increase is followed by a short-lasting pulse of southward interplanetary magnetic field. The circulation of solar plasma changes, and the electron energy gain results in fluxes of energetic electrons observed at dawn.

As the method gives an insight into the details of a single particle motion, attention is focused on electrons involved in

Published by Copernicus Publications on behalf of the European Geosciences Union. 
Table 1. Plasma parameters and the object radius.

\begin{tabular}{ccccc}
\hline$n_{o}\left[\mathrm{~m}^{-3}\right]$ & $B_{\mathrm{SW}}[\mathrm{nT}]$ & $V_{\mathrm{SW}}[\mathrm{km} / \mathrm{s}]$ & $B_{O}[\mathrm{nT}]$ & $r_{a}[\mathrm{~km}]$ \\
\hline $3 \times 10^{4}$ & $\leq 2.8$ & 300. & 10. & 2000. \\
\hline
\end{tabular}

the processes and the history of their characteristics is presented. In this way, it is demonstrated that the PIC method reproduces processes typical for magnetospheres and gives the finest details of electron dynamics. The layout of the paper is as follows: in the next section the magnetosphere and the wind are defined in detail, the governing equations are presented and numerical parameters in solving the equations are specified. The results of the calculations of the evolution of the \{object, wind system are presented in Sects. 3 and 4. In Sect. 5 the results are discussed and compared with the corresponding experimental data. Section 6 concludes the paper.

\section{The definition of an \{object, wind $\}$ system, governing equations and simulation details}

An object of a radius of $r_{a}=2 \times 10^{6} \mathrm{~m}$ is considered. It is weakly magnetized; the magnetic induction at the equator $B_{o}$ is $10 \mathrm{nT}$. The magnetic moment direction is southward. The object has neither ionosphere, nor the closure of magnetospheric currents in the ground. The wind particles interaction with the object surface is not incorporated in the model. As in the original paper by Buneman (1993), the temporal evolution of the system is governed by an equation of particle motion and by Maxwell's equations describing the change of electric and magnetic fields. The equations, in mksA units, read:

$\frac{d \mathbf{v}}{d t}=\frac{Q}{M}(\mathbf{E}+\mathbf{v} \times \mathbf{B})$

$\frac{d \mathbf{r}}{d t}=\mathbf{v}$

$\frac{\partial \mathbf{B}}{\partial t}=-\nabla \times \mathbf{E}$

$\varepsilon_{o} \frac{\partial \mathbf{E}}{\partial t}=\mu_{o}^{-1} \nabla \times \mathbf{B}-\mathbf{j}$

where: $\mathbf{v}$ and $\mathbf{r}$ stand for velocity and position of a particle of charge $Q$ and of mass $M$. The electric and magnetic fields are denoted by $\mathbf{E}$ and $\mathbf{B}$, respectively, and $\varepsilon_{o}, \mu_{o}$ designate the dielectric constant and the magnetic permeability of vacuum. If interested, the reader may consult Buneman's paper for the adapted boundary conditions for fields and particles. Resolution in time and in space, the size of simulation domain, grid of points and plasma macroparticles - these parameters are re-defined in this study. So, the time step is
$\Delta t=0.004 \mathrm{~s}$, space resolution is $\Delta x=0.1 r_{a}$, the domain size is $25.6 \times 25.6 \times 25.6 r_{a}^{3}$, and the celestial body is at the centre of the cube, $\left(x_{o}, y_{o}, z_{o}\right)=(12.85,12.85,12.8) r_{a}$. The grid of $256 \times 256 \times 256$ points is regular. The macroparticle mass and charge are determined by the number density of the real plasma. The equations are normalized and solved for computer quantities corresponding to position, velocity, current density, electric field and magnetic induction. However, the results are presented in mksA units.

The particles move freely in the simulation domain. Their contribution to the current density $\mathbf{j}$, is calculated in a way to satisfy the charge conservation law (Villasenor and Buneman, 1992), and the method requires the maximum value of particle speed be less than $0.5 \Delta x / \Delta t$. In one step of the time loop, particles are pushed, the current density term is calculated and the fields are updated. The most restrictive numerical stability condition concerns the time step an electron may be traced with: $\Delta t \leq 2 /\left(Q_{e} B / M_{e}\right)=2 / \Omega_{e}$, and those regions where $\mathrm{B}$ is too high to satisfy the condition are closed for particles. In contrast to the original formulation, the proton to electron mass ratio is real: $M_{p} / M_{e}=1836$.

The simulation is preceded by two auxiliary runs of the code. First, the dipole field evolves in the domain voided of plasma in order to allow the spurious transients to evanesce. Then the wind begins to enter the domain. Electron/proton pairs cross the front wall and the injection regime is such that, on average, there is one electron/proton pair per cell in the wind flow. The wind is quiet, its bulk velocity is $v_{\mathrm{SW}}=0.15 r_{a} / s$, thermal velocity of electrons $v_{\text {the }}=v_{\mathrm{SW}}$. The fields propagate with velocity $c=v_{\mathrm{SW}}$. The second run lasts $180 \mathrm{~s}$. The domain, filled with fields and plasma, is ready to be disturbed by an arbitrary event. That is the moment $t=0$.

The ranges of plasma standard characteristics - electron plasma oscillation frequency, electron cyclotron frequency, thermal cyclotron radius, and magnetic pressure - follow from the values of these three quantities: number density, magnetic induction intensity and electron thermal velocity. The values of the solar wind number density, magnetic induction intensity and velocity, as well as the values characterising the celestial body - the intensity of magnetic induction at the equator and the radius - are written in Table 1.

Consequently, the electron plasma oscillation frequency $\omega_{p e}=56 \sqrt{n_{o}}>9700 \mathrm{rad} / \mathrm{s}$, the electron cyclotron frequency $\Omega_{e}=e B / m_{e}<498 \mathrm{rad} / \mathrm{s}$, if $B$ is smaller than $2.8 \mathrm{nT}$. The thermal cyclotron radius, $\rho_{L} / r_{a}=v_{\text {the }} / \Omega_{e} / r_{a}>3 \times 10^{-4}$, and the magnetic pressure $B^{2} / 2 \mu_{o}<3.1 \times 10^{-12} \mathrm{~Pa}$. Number density unit is $n_{o}=3 \times 10^{4} \mathrm{~m}^{-3}$. Energy density unit is defined as $n_{o} \times 1 \mathrm{eV}=30 \mathrm{keV} / \mathrm{m}^{3}$. This is the system \{magnetized object, solar wind investigated in the study.

In Fig. 1. the distribution of electron number density in the equatorial plane at the commencement of the simulation, at $t=1 \mathrm{~s}$, is presented. The narrow parabolic region of elevated number density (up to 4 macroelectrons/cell, and number density $12 \times 10^{4} \mathrm{~m}^{-3}$ ) represents the magnetopause. Its distance to the body surface depends on the local time; at 


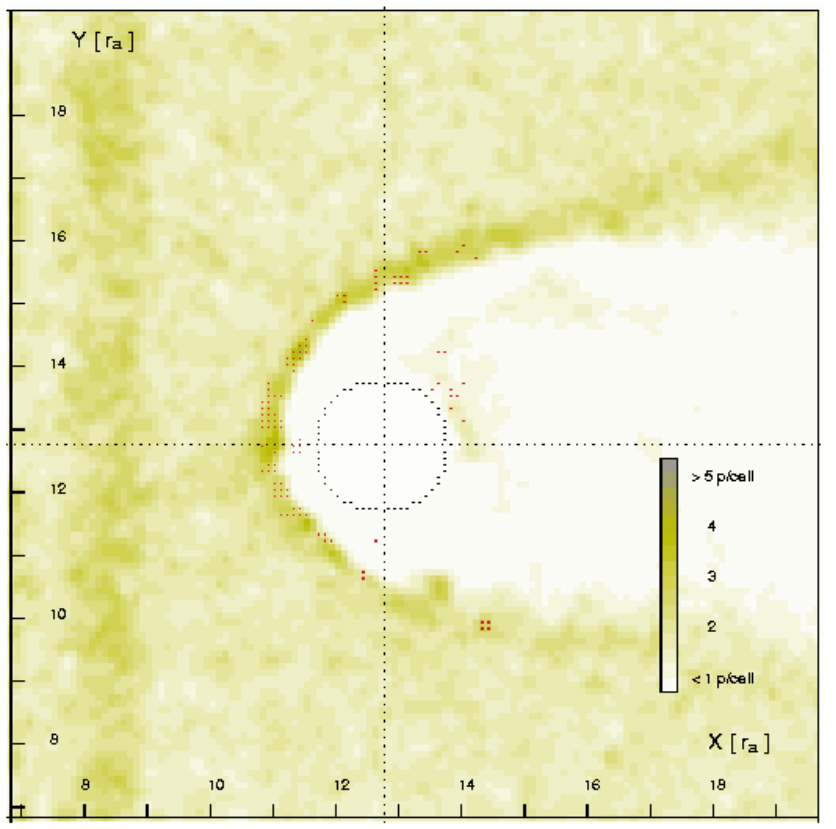

Fig. 1. Distribution of the number density of macroelectrons in the equatorial plane, at $t=1 \mathrm{~s}$. The grid points where the current density is above $9 \mathrm{nA} / \mathrm{m}^{2}$ level, are marked in red. Number density unit is $n_{o}$.

noon it is $0.6 r_{a}$. The red dots represent grid points at which the current density is above the level of $9 \mathrm{nA} / \mathrm{m}^{2}$. Near the front wall on the left there is a dense surge of electrons moving towards the magnetosphere. In Fig. 2. the distribution of magnetic induction intensity in the noon-midnight plane at $t=1 s$, is presented. The magnetic field lines, except those lying beyond the plane, are overplotted in black. Lines are traced for several latitudes at every $5^{\circ}$, beginning at lat $=15^{\circ}$. As in the previous figure, the grid points where the current density is high are plotted in red. In the next $20 \mathrm{~s}$ the density surge will reach the magnetopause resulting in the events described in the following section.

\section{Results of simulations; wave activity}

As the surge reaches the magnetopause, the level of current density fluctuations increases and waves are generated. The process is followed by: observing the distribution of the $B_{z}$ component at several points in the equatorial plane, analysing the spectral content of the multi-point signal, and following an electron and the fields it encounters on its course.

In Fig. 3. the equatorial distribution of the $B_{z}$ component in the noon-dusk sector at $t=20 \mathrm{~s}$ is colour coded. The majority of the region presented is amorphous and $B_{z}$ is at a near zero level. At the edge of the region where the component is positive, that is at the external border of the magnetopause, spots of negative $B_{z}$ are discernible. Their shape

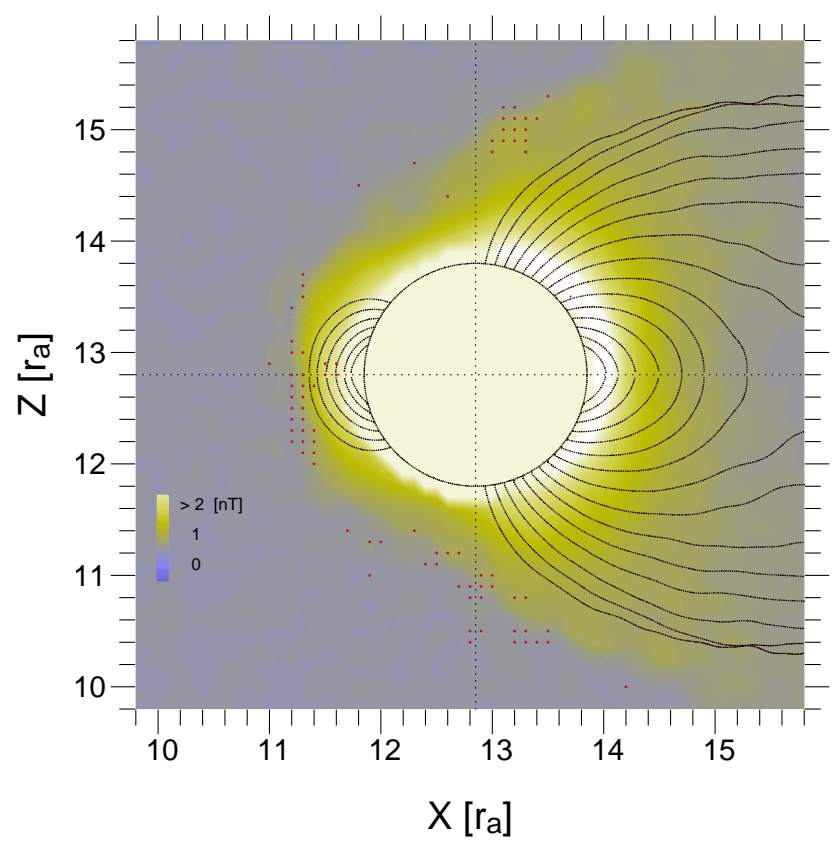

Fig. 2. Distribution of the magnetic induction intensity, $B$ in the noon-midnight plane, at $t=1 \mathrm{~s}$. Magnetic field lines are overplotted in black and grid points, where the level of the current density is above $9 \mathrm{nA} / \mathrm{m}^{2}$, are plotted in red.

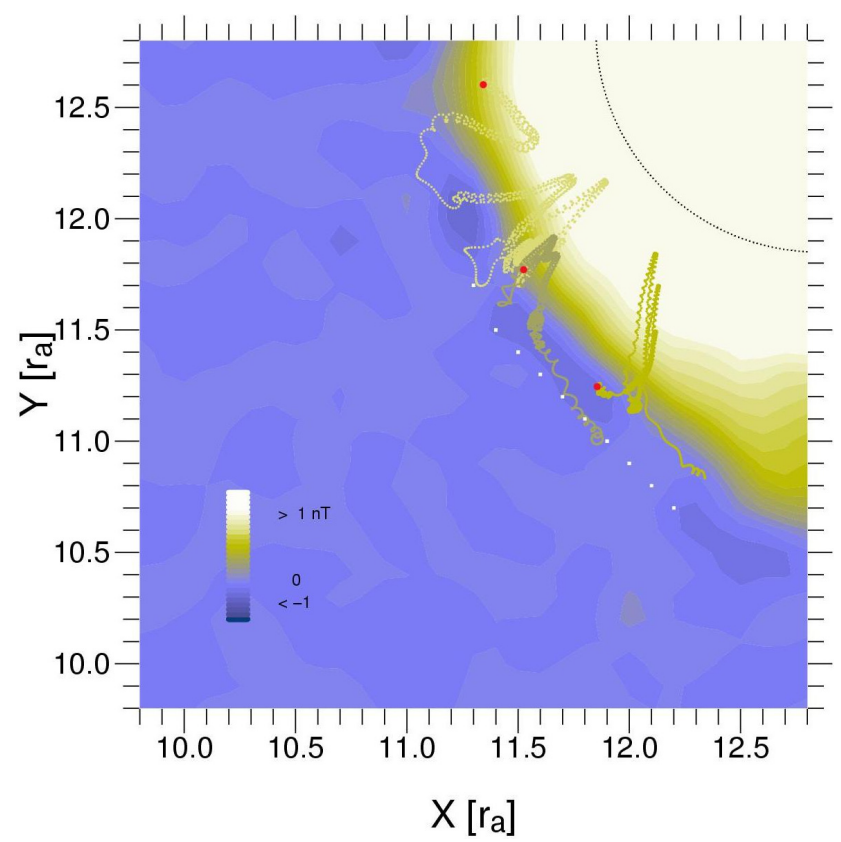

Fig. 3. Distribution of the Z-component of the magnetic induction in $(x, y)$ plane, near the object and its magnetopause. Parts of three electron trajectories are overplotted, commencing with red points, and the wave duct is represented by the train of ten white points. 


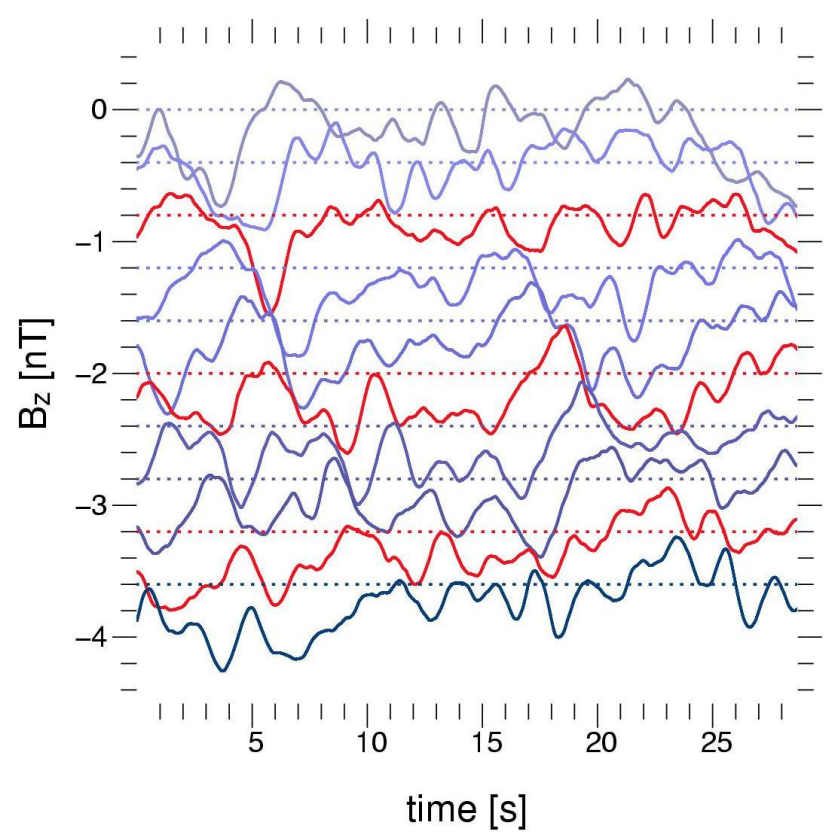

Fig. 4a. $B_{z}$ component profiles recorded at the points in the wave duct, plotted in white in Fig. 3. Except for the top profile, recorded at a point near noon, the following records and the corresponding zero-level lines, have been shifted by $-0.4 \times N \mathrm{nT}$, for legibility $(N=0, \ldots, 9)$.

is elongated in local time and they propagate from noon towards dusk. The propagation is illustrated in Fig. 4a, where the time profiles of the component recorded at those points plotted in white in Fig. 3, are presented. The deep depression in $B_{z}$, down to the level of $-1 \mathrm{nT}$ followed by oscillations of a smaller amplitude is characteristic for each profile. What is the spectral content of the signal?

The spectrum is calculated in a dynamic way; a $4 \mathrm{~s}$ window is used to observe the changes in the spectral content of the signal. The spectrum of the signal recorded at point $(x, y)=(11.5,11.4) r_{a}$ (the first profile in red in Fig. 4a) is presented in Fig. $4 \mathrm{~b}$ in two forms. In the inlet in the top right, the ranges of the normalised power spectral density (PSD) for each frequency are plotted. The first resolved frequency is $f_{1}=0.25 \mathrm{~Hz}$, next are multiples of $f_{1}$, and the spectrum is contained in the range up to $f=3 \mathrm{~Hz}$. The electron cyclotron frequency range is $f_{c}<3 \mathrm{~Hz}$, and multiples of $0.1 f_{c}$ are present in the spectrum. This is characteristic for whistler family waves. In the main part of Fig. 4b, the components of the spectrum (preserving the colour assignement in the inlet) are plotted versus time to illustrate the varying spectral content of the signal due to the complex wave passing the point.

Another feature of the fields excited in the region may be seen while observing the electrons. In Fig. 3 there are three examples of electron trajectories. Let us follow the electron near dusk during $6 \mathrm{~s}$ of motion. In Fig. 5a, the trajectory is

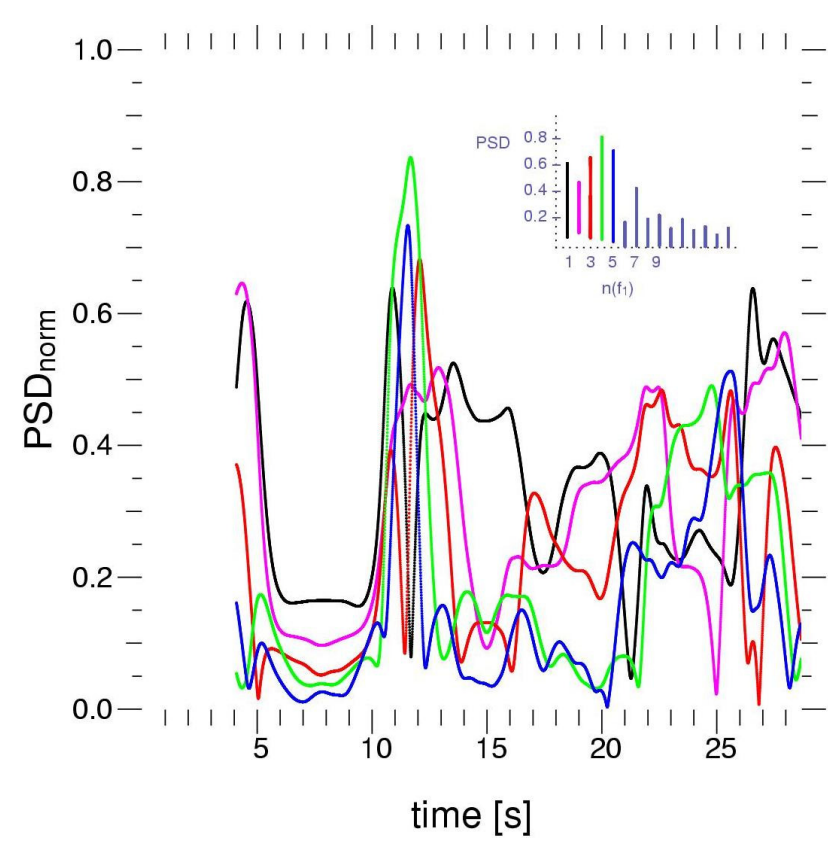

Fig. 4b. Spectral analysis of the $B_{z}$ signal, recorded at the point (x, y) $=(11.5,11.4) r_{a}$, and plotted in red in Fig. $4 \mathrm{a}$. In the top right, the spectrum accumulated during $3 \mathrm{~s}$ is shown and below, the corresponding spectral components intensities are plotted versus time.

Table 2. Time, energy, position and magnetic induction along the dusk- electron trajectory.

\begin{tabular}{lcccrrc}
\hline$t[\mathrm{~s}]$ & $\epsilon[\mathrm{keV}]$ & $\rho\left[r_{a}\right]$ & $\mathrm{Z}\left[r_{a}\right]$ & $B_{x}[\mathrm{nT}]$ & $B_{y}[\mathrm{nT}]$ & $B_{z}[\mathrm{nT}]$ \\
\hline 0. & 0.019 & 1.87 & 12.86 & -0.147 & 0.095 & 0.276 \\
1.72 & 0.020 & 1.36 & 11.93 & -0.220 & -0.950 & 0.745 \\
2.35 & 0.017 & 1.83 & 12.70 & -0.092 & -0.129 & 0.549 \\
3.02 & 0.036 & 1.54 & 12.15 & -0.128 & -0.770 & 0.840 \\
3.95 & 0.013 & 1.92 & 12.97 & -0.075 & 0.009 & 0.292 \\
5.41 & 0.041 & 1.24 & 13.74 & 0.306 & 1.012 & 0.454 \\
\hline
\end{tabular}

drawn in $(\rho, z)$ plane ( $\rho$ is the cylindrical radius). Energies, positions of the electron and magnetic field components at the commencement (first row) and at five consecutive moments are presented in Table 2. These moments are marked with vertical lines in Fig. 5b, where electron energy, position, electric and magnetic fields and the cosine of the angle between the fields are plotted versus time.

At $t=0$, the electron is in the equatorial plane. Its energy is $20 \mathrm{eV}$ and it begins to bounce along a magnetic filed line, which crosses the equatorial plane at $\rho=1.8 r_{a}$. At the mirror points on this line - at $z=11.93 r_{a}$ and at $z=13.74 r_{a}$ - the electron experiences the strongest electric field, at the level of $0.15 \mathrm{mV} / \mathrm{m}$. The E-field there is oriented downward to the object surface (the sign of $\cos \angle(\mathbf{E}, \mathbf{B})$ is -1 in the south and +1 in the north). Consequently, the electron loses energy while approaching a miror point $(d($ energy $) / d t=q / \mathrm{m}(\mathbf{E} \cdot \mathbf{v})$, $q<0)$. 


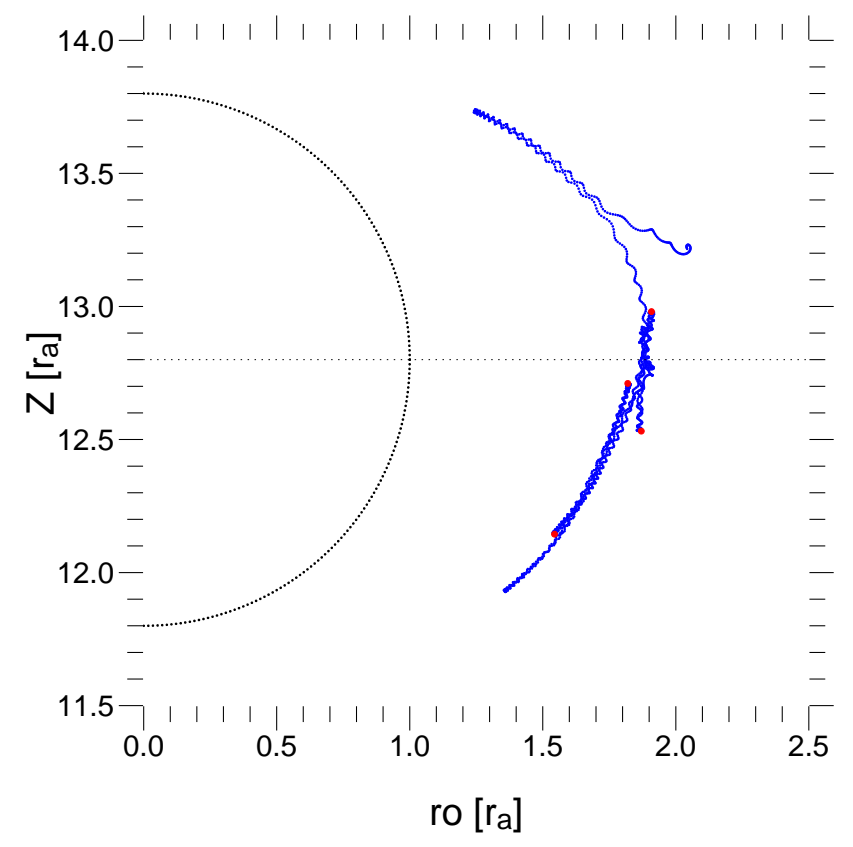

Fig. 5a. Dusk-electron trajectory in $(z, \rho)$ plane, where $\rho$ is the cylindrical radius, $\rho=\sqrt{\left(x-x_{o}\right)^{2}+\left(y-y_{o}\right)^{2}}$. The moments $t=2.352,3.02,3.953,4.52 \mathrm{~s}$ are marked in red. The surface of the object is represented by a circle.

Four additional "mirroring", at $t=2.35,3.02,3.95,4.52 \mathrm{~s}$ are the result of fields associated with waves discussed earlier. The deep depression of $B_{z}$ in the profiles in Fig. $4 \mathrm{a}$ and the oscillations that follow it, imprinted in the dipole field, modify the electron motion. Here, the attention is focused on the z-component of the motion, governed by the equation: $d v_{z} / d t=q / m\left(E_{z}+v_{x} B_{y}-v_{y} B_{x}\right)$. As $v_{x}, v_{y}$ are at the level of hundreds of $\mathrm{km} / \mathrm{s}$, and $B_{x}$ and $B_{y} \leq 0.1 \mathrm{nT}, E_{z}$ prevails over the smaller terms of the equation. So, $d v_{z} \approx \int E_{z} d t$ and $z \approx \int v_{z} d t$. At local extrema of z-coordinate, $v_{z}$ vanishes. A local minimum of this velocity component at $t=3.4 \mathrm{~s}$ marks the moment when the electron begins an oscillation, finished at $t=4.7 \mathrm{~s}$ when it returns to bouncing. The period of oscillation is estimated as the time between the moments, where $z-z_{o}=0: \tau=4.74-3.74=1 \mathrm{~s}$, so the frequency $f=1.0 \mathrm{~Hz}$. The magnetic induction is at the level of $0.425 \mathrm{nT}$, and $f_{c}=12 \mathrm{~Hz}$. So, the oscillation frequency $f \approx 0.1 f_{c}$, and the wave is one from the whistler family. Neglecting the propagation effect, one has: if $E_{z} \approx-\cos 2 \pi f t$, then $v_{z} \approx-q \sin 2 \pi f t$, and $z \approx-\cos 2 \pi f t$.

\section{Results of simulations; interplanetary fields effect}

What was described in the previous section was the effect of a narrow layer of dense plasma on the magnetosphere. In this section the effect of interplanetary electromagnetic

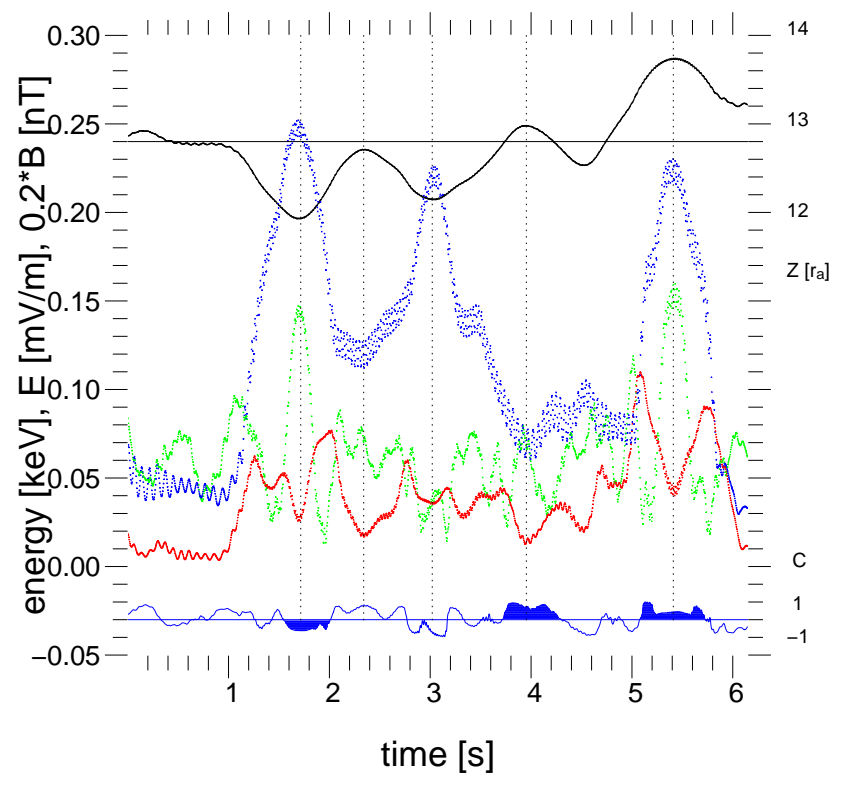

Fig. 5b. Time profiles of the dusk-electron energy (in red), and the fields it encounters, electric (in green) and magnetic (in blue). The curve $\mathrm{C}$ in blue situated below the profiles at -0.03 level, is the cosine of the angle between the two fields. The curve in black above the profiles is the z-coordinate of the electron position. Scales for the two are on the right.

fields is described. The disturbance is composed of the dense layer and a short-lasting ( $2 \mathrm{~s})$ impulse of an electric field, $\mathbf{E}=-\partial \mathbf{A} / \partial t=-d f / d t \mathbf{A}$, the source of which is a remote dipole. Maxwell's equation $\partial \mathbf{B} / \partial t=-\nabla \times \mathbf{E}$ gives the associated magnetic field $\mathbf{B}=\left(0,0, B_{z}\right), B_{z}=-2.5 \mathrm{nT}$ at $x=6.4 r_{a}$. The fields propagate towards the object. The energy of the plasma increases, and the velocity field pattern is modified. Attention is focused on one aspect of the process: the identification of the source of energetic $(1 \mathrm{keV})$ electrons populating a near-equatorial region at dawn, at a radial distance of $r>0.5 r_{a}$ above the surface.

In Fig. 6, which presents the equatorial distribution of the electron energy density at $t=46 \mathrm{~s}$ ( $4 \mathrm{~s}$ before the end of simulation), the region of concern is easily discernible. Average equatorial velocity vectors of electrons are overplotted (instead of using arrows which take up too much space, the vector ends are represented by bold red dots). The energy density, which is most intense in the interval $x=7-8 r_{a}$ becomes extinct as the distance to the object becomes less. This results from the fact that the finite amount of the energy brought by the fields is distributed throughout the particles. Their energy increases and some of them populate the dawn equatorial region, moving westward. However, a far greater contribution of energetic electrons to the region comes from the nightside, due to the substorm-like collapse; the open high-latitude magnetic field lines close and move to the dayside. The $V_{y^{-}}$ positive velocity field associated with the re-configuration, 


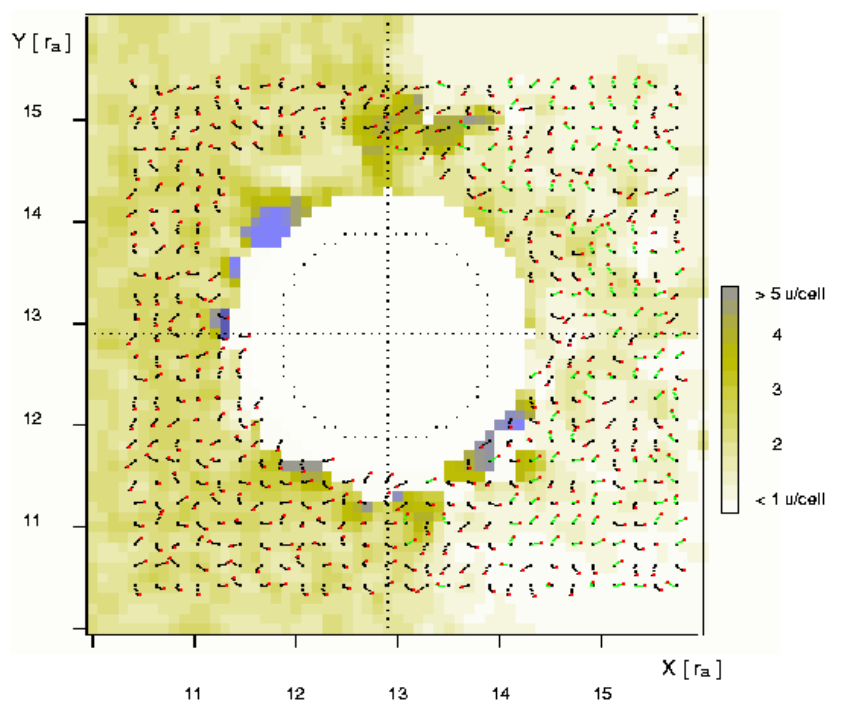

Fig. 6. Distribution of the energy density in the equatorial plane at $t=46 \mathrm{~s}$. The electron velocity field $\left(V_{x}, V_{y}\right)$ is overplotted. Each vector is represented by dots - the vector commences with a bold black dot and ends with a bold red one. The night-side velocity vectors of positive y-component are displayed in green. Energy density unit is $30 \mathrm{keV} / \mathrm{m}^{3}$.

is marked by green vectors; in the aftermidnight sector it is dawnward (eastward). Positions of ten electrons representing the group, plotted every second, are shown in the next two figures; in Fig. $7 \mathrm{a}$ - the projection on the $(x, y)$ plane, and in Fig. $7 \mathrm{~b}-$ the $(y, z)$ projection. The initial positions are marked by red dots and the lapse of time is colour coded according to the color bar at the top of Fig. 7a. The crescentshaped region filled with energetic $(1 \mathrm{keV})$ electrons is localised near 05:00 LT.

The electron energy change in time is illustrated in Fig. 8, where again colour is used to indicate the time. Except for two electrons, which begin their motion in the region of elevated electric field intensity (above $0.38 \mathrm{mV} / \mathrm{m}$ ), the majority of the group starts in a region of weak electric field intensity - below $0.1 \mathrm{mV} / \mathrm{m}$, with energies at a level below $1 \mathrm{keV}$.

\section{Discussion of results}

The global structure of the investigated magnetosphere of a celestial body is discussed referring to the studies of Simon et al. (2006), and of Omidi et al. (2002, 2003).

Simon et al. (2006) simulated the plasma environment of a magnetized asteroid using a 3-D hybrid code where the plasma is represented by macroprotons and an electron fluid. The authors elucidated the details of the motion of protons traversing the interaction region - a boundary layer between the solar wind and inner regions of the magnetosphere - and have illustrated their dependence on the direction of the in-

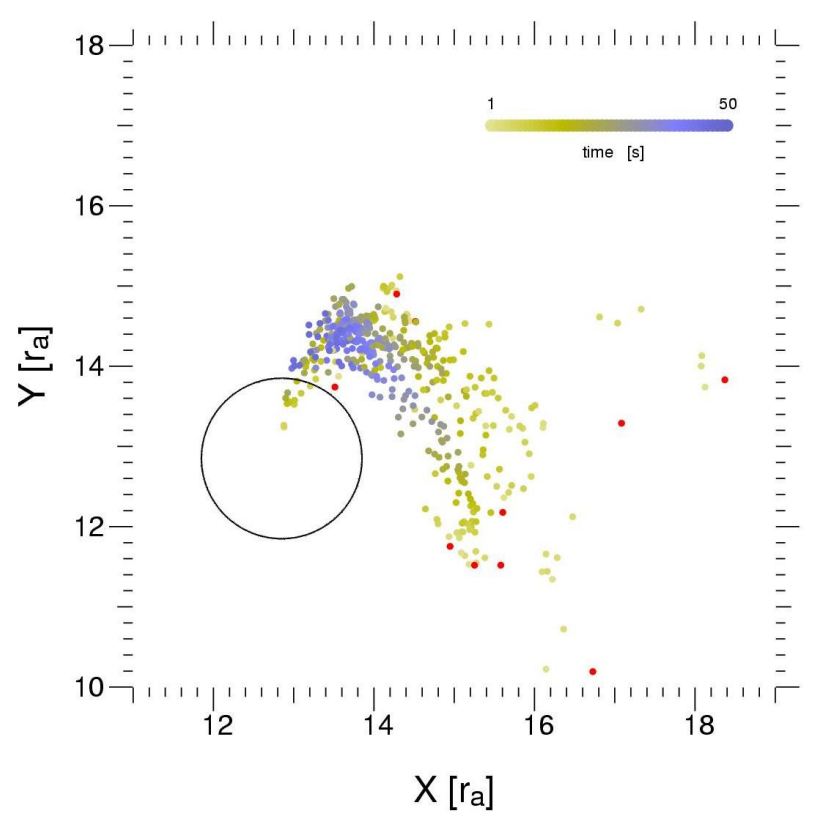

Fig. 7a. (x, y) positions of the electrons populating the region at dawn, where the level of energy density is high - above 4 units $\left(120 \mathrm{keV} / \mathrm{m}^{3}\right)$. The points are plotted every second, and the elapsing time is represented by colour according to the scale at the top.

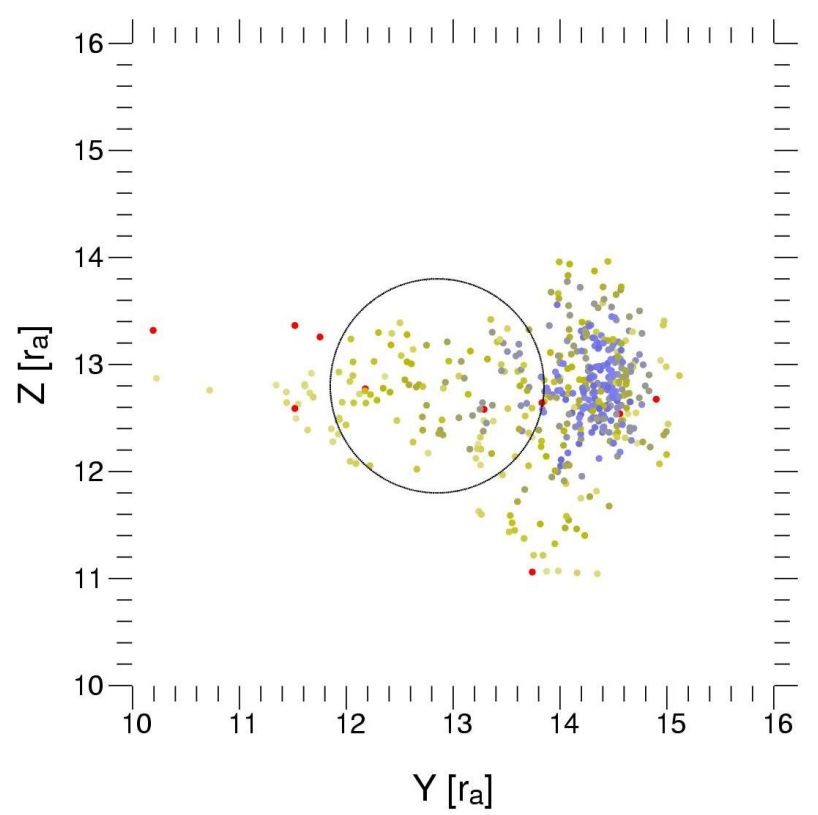

Fig. 7b. (y, z) positions of the dawn electrons plotted in Fig. 7a.

terplanetary magnetic field. The values of quantities defining that system are in Table 3.

Resolution in space is $\Delta x=0.25 r_{a}$, and in time $\Delta t=0.2 \mathrm{~s}$. Though differences exist (compare Tables 1 and 3), the two 
Table 3. Plasma parameters and the object radius.

\begin{tabular}{ccccc}
\hline$n_{o}\left[\mathrm{~m}^{-3}\right]$ & $B_{\mathrm{SW}}[\mathrm{nT}]$ & $V_{\mathrm{SW}}[\mathrm{km} / \mathrm{s}]$ & $B_{o}[\mathrm{nT}]$ & $r_{a}[\mathrm{~km}]$ \\
\hline $1.4 \times 10^{6}$ & 2.7 & 400. & 392. & 1365. \\
\hline
\end{tabular}

objects do have a poorly structured magnetopause boundary layer as compared with the magnetosphere of the Earth. The shock-like layer is reduced, in the study of Simon et al. (2006), to a thin coat where the number density maximizes. In the case of the weakly-magnetized body considered in this study, the thin layer of elevated number density of plasma is not observed.

The terrestrial magnetosphere was the magnetosphere of reference in the study of Omidi et al. (2003). The authors simulated dipolar magnetospheres of different magnetic moments, using a 2-D hybrid code. The aim of that study was to find a critical value of the stand-off distance, a distance from a magnetic obstacle centre upstream to the point $r^{*}$ in the solar wind, where the solar wind dynamic pressure is balanced by the magnetic pressure of the obstacle's field: $n_{o} m_{p} V_{\mathrm{SW}}^{2}=B_{o}^{2} /\left(r^{*} / r_{a}\right)^{6} / 2 \mu_{o}$. When expressed in the object radius, the distance is denoted by $R_{\mathrm{off}}=r^{*} / r_{a}$. The authors expressed the stand-off distance in proton inertial length $\lambda_{i p}=c / \omega_{p p}$, where $c$ stands for light velocity in vacuum, and $\omega_{p p}$ denotes the proton plasma frequency. They found that $D_{p}=r^{*} / \lambda_{i p}$ must be greater than twenty for a magnetosphere to be of a terrestrial type. So, the results discussed do not contradict the result of Omidi et al. (2003): $D_{p}=16.4$ for the asteroid investigated by Simon et al. (2006) and $D_{p}=2.4$ for the weakly-magnetized object considered in this study. Neither magnetosphere is terrestrial-like.

The presence of waves from the whistler branch $(f=(0.1-$ $0.8) f_{c}$ ) in the vicinity of a weakly-magnetized body immersed in a low density wind, is not unusual and should have been expected. The waves have been observed in external regions of the terrestrial magnetosphere, near Mars, Venus, Mercury, asteroids (Greenstadt, 1997), and near lunar crustal magnetic sources (Halekas et al., 2006). They have been studied since the late sixties, and simulated recently by Sydora et al. (2007). In our simulations the number of particles per cell is too low to reliably estimate the electron velocity distribution function, and the temperature anisotropy. Therefore, the specific characteristics of plasma favorables to the existence of observed waves are presented.

In a comprehensive study of Dubinin et al. (2007), CLUSTER observations of whistler emissions at the dusk edge of the terrestrial magnetosphere are reported and explained theoretically. The authors refer to the idea of cyclotron resonant instability as the cause of a whistler wave. Resonant electrons are necessary to trigger the cyclotron resonant instability and excite a whistler wave. The energy of these electrons is above a threshold value $E^{*}$, which depends on the

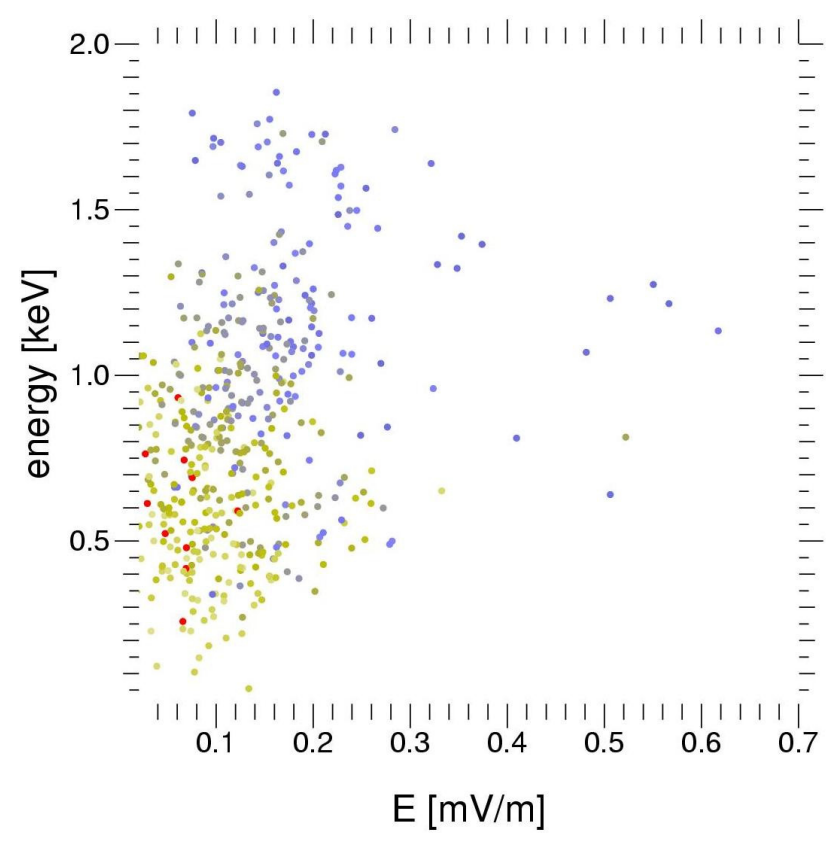

Fig. 8. Energy of the dawn energetic population of electrons and the associated electric field intensity in time. Color coded, as in Fig. 7a.

strength of magnetic induction, on the number density and the wave frequency: $E^{*}=B^{2} / 2 \mu_{o} n_{o}\left(1-\omega / \omega_{c e}\right)^{3} \omega_{c e} / \omega$. Are there any resonant electrons in the region where waves are observed? In the plasma near the weakly- magnetized object ( $\left.B=0.4 \mathrm{nT}, n_{o}=3 \times 10^{4} \mathrm{~m}^{-3}\right), B^{2} / 2 \mu_{o} n_{o}=13 \mathrm{eV}$. The range of the frequency factor $\left(1-\omega / \omega_{c e}\right)^{3} \omega_{c e} / \omega$ is $0.54-7.29$, if $\omega / \omega_{c e}$ ranges from 0.1 to 0.4 . Electrons of the energy $E>E^{*}$ are present in the local time sector between noon and dusk, near the magnetopause i.e. in the wave region.

In a series of papers Stasiewicz proposed (see for example 2005, 2006 papers) a two-fluid theory of linear and nonlinear magnetohydrodynamic waves and presented its applications. The dispersion diagrams in the form of the wave number expressed in ion inertial length units as a function of two variables - the Alfvén Mach number and the angle between the wave vector and the magnetic field - are built for a wide range of plasma parameters. The diagram presented in Fig. 2 of the 2006 paper concerns the very plasma in the region where waves are observed in this study. Assuming $n_{o}=3 \times 10^{4} \mathrm{~m}^{-3}$ and $B=0.4 \mathrm{nT}$, the Alfvén velocity $V_{A}=B / \sqrt{\mu_{o} n m_{p}}=5 \times 10^{4} \mathrm{~m} / \mathrm{s}$. The phase velocity may be estimated from Fig. $4, V_{f}=0.6 r_{a} / 2 \mathrm{~s}=6 \times 10^{5} \mathrm{~m} / \mathrm{s}$. So, the Alfvén Mach number $M_{A}=12$. Plasma beta $\beta=n m_{e} v_{\text {the }}^{2} / B^{2} / 2 \mu_{o}>0.017$, if $v_{\text {the }}>0.1 r_{a} / s$. The diagram computed for those parameters does not change; the nonlinear whistler branch still fills the region of large Mach numbers and does not cover the small angles between the wave vector and magnetic field direction. The wave length may be estimated: $\lambda<0.84 \times 10^{6}=0.42 r_{a}$ as $\log \left(k \lambda_{i}\right)>1$, and the ion 
inertial length $\lambda_{i}=c / \omega_{p i}=1.35 \times 10^{6}$. The diagram for nonlinear waves in such a plasma in Fig. 2 of Stasiewicz's (2006) paper, supports the idea, the waves described in Sect. 3 belong to the nonlinear whistler branch. Whistlers' presence at the magnetopause edge is an immanent feature of magnetospheres of which the investigated magnetosphere is not deprived.

During the first encounter with Mercury, 35 years ago, on 29 March 1974, MARINER 10, on the inbound path of its trajectory, detected whistler mode waves, crossed the bow shock and the magnetopause, and entered the region where the x-component of magnetic induction dropped far below the dipole level, $B_{x}<-80 \mathrm{nT}$ (see for example McKennaLawlor, 1997). This was associated with the presence of a current in the tail (Whang, 1977). An hour later, on the outbound path, near the magnetopause two peaks of counts of $300 \mathrm{keV}$ electrons were detected, suggesting the space-craft crossed a specific region populated by an energised electron component. The data were analysed and interpreted by Christon (1987). The author compared hermean and terrestrial data from analogous regions, in terms of electron energy distribution. In both cases it was a kappa distribution - a Maxwellian core and high energy tail. The events were associated with multiple onsets during a hermean substorm which appeared to be about 30 times shorter than the terrestrial substorms. In the case of a weakly-magnetized object, the analogous region is populated by electrons of energy above $1 \mathrm{keV}$ level, in two distinct components - that coming from the dayside is dominated by one coming from the nightside. The latter is associated with a collapse of the magnetosphere after the onset of a substorm-like event. The time-scale of the event is less than a minute.

\section{Conclusion}

A modified version of TRISTAN was applied to simulate a weakly- magnetized celestial body of Mercury's size immersed in a solar wind of low density. High resolution in time (time step $\Delta t=0.004 \mathrm{~s}$ ) allows to avoid numerical instability while tracing electrons. An electron-scale model of a magnetosphere has been built, and enables the following concluding statements to be made.

1. Referring to the sequence of magnetospheres, considered by Omidi et al. (2004), the results of the study support the conjecture that objects weakly magnetized, if immersed in a solar wind of low density, may have a magnetosphere. It is small, variable and not of a terrestrial type. The stand-off distance expressed in ion inertial length is below the corresponding value for the Earth: $D_{p}=2.4<20$. The magnetic field lines which are anchored at latitudes greater than $25^{\circ}$ are easy to open and wave in the wind, changing the shape and the position of surfaces they span. The life time of a configu- ration of the magnetosphere is short, as short as tens of seconds.

2. The magnetospheric processes described in the study correspond to known and understood phenomena in solar system magnetospheres; magnetohydrodynamic waves are excited at the edge of the magnetopause, and energetic particles associated with a substorm-like event are observed at dawn.

3. The results demonstrate that the PIC code is able to reproduce the two magnetospheric processes. Moreover, the code, if applied with care, gives an insight into the kinetic details of the motion of electrons, as well as into the fine structure of electric and magnetic fields.

4. To establish correspondence of results with reality, measurements related to the processes, made on board of space probes have been referred to. The study initiates the observations of magnetospheric macroparticles, applying parallel PIC methods. This requires large computer resources but the authoress believes this barrier is short-lived.

Acknowledgements. Calculations were partly conducted using computers at the Interdisciplinary Centre for Mathematical and Computational Modelling at Warsaw University. The authoress is grateful to the staff of the Centre for being introduced into parallel calculus. Thanks go to David H. Munro for YORICK - the visualization language used to construct figures. I do appreciate the linguistic help of Graham Carr. The study was supported in the frame of a French-Polish CNRS-PAN project No. 16674 and the hospitality during the visits at CETP-CNRS in Saint-Maur is much appreciated.

Topical Editor I. A. Daglis thanks K.-I. Nishikawa and another anonymous referee for their help in evaluating this paper.

\section{References}

Buneman, O.: TRISTAN. The 3D Electromagnetic Particle Code, in: Computer Space Plasma Physics: Simulation Techniques and Software, edited by: Matsumoto, H. and Omura, Y., TERRA, Tokyo, 1993.

Blanco-Cano, X., Omidi, N., and Russel, C. T.: Hybrid simulation of solar wind interaction with magnetized asteroids: Comparison with Gallileo observations near Gaspra and Ida, J. Geophys. Res., 108(A5), 1216, doi:10.1029/2002JA009618, 2003.

Cai, D., Yan, X. Y., Nishikawa, K.-Y., and Lembege, B.: Particle entry into the inner magnetosphere during duskward IMF $B_{y}$ : Global three-dimensional electromagnetic full particle simulations, Geophys. Res. Lett., 33, L12101, doi:10.1029/2005GL023520, 2006.

Christon, S. P.: A Comparison of the Mercury and Earth Magnetospheres: Electron Measurements and Substorm Time Scales, Icarus, 71, 448-471, 1987.

Delcourt, D. C., Moore, T. E., Orsini, S., and Millilo, A., and Sauvaud, J.-A.: Centrifugal acceleration of ions near Mercury, Geophys. Res. Lett., 29(12), 1591, doi:10.1029/2001GL013829, 2002. 
Dubinin, E. M., Maksimovic, M., Cornilleau-Wehrlin, N., Fontaine, D., Travnicek, P., Mangeney, A., Alexandrova, O., Sauer, K., Fraenz, M., Dandouras, I., Lucek, E., Fazakerley, A., Balogh, A., and Andre, M.: Coherent whistler emissions in the magnetosphere - Cluster observations, Ann. Geophys., 25, 303-315, 2007, http://www.ann-geophys.net/25/303/2007/.

Greenstadt, E. W.: Conditions for Magnetic Interaction of Asteroids with the Solar Wind, Icarus, 14, 374-381, 1997.

Halekas, J. S., Brain, D. A., Mitchell, D. L., and Lin, R. P.: Whistler waves observed near lunar crustal magnetic sources, Geophys. Res. Lett., 33, L22104, doi:10.1029/2006GL027684, 2006.

Ip, W. H. and Kopp, A.: MHD simulations of the solar wind interaction with Mercury, J. Geophys. Res., 107(A11), 1348, doi:10.1029/2001JA009171, 2002.

Kabin, K., Gombosi, T. I., DeZeeuw, D. L., and Powell, K. G.: Interaction of Mercury with the solar wind, Icarus, 143, 397406, 2000.

Kallio, E. and Janhunen, P.: Modelling the solar wind interaction with Mercury by a quasi-neutral hybrid model, Ann. Geophys., 21, 2133-2145, 2003,

http://www.ann-geophys.net/21/2133/2003/.

Kivelson, M., Bargatze, L. P., Kurana, K. K., Southwood, D. J., Walker, R. J., and Colemann Jr. P. J.: Magnetic Field Signatures Near Galileos Closest Approach to Gaspra, Science, 261, 331334, 1993.

McKenna-Lawlor, S. M. P.: Characteristic boundaries of the hermean magnetosphere and energetic particles close to the planet, Planet. Space Sci., 45, 167-180, 1997.

Nishikawa, K.-I.: Particle entry into the magnetosphere with a southward interplanetary magnetic field studied by a threedimensional electromagnetic particle code, J. Geophys. Res., 102(A8), 17631-1741, 1997.

Nishikawa, K.-I.: Global particle simulation study of substorm onset and particle acceleration, Space Sci. Rev., 95, 361-371, 2001.

Omidi, N., Blanco-Cano, X., Russel, C. T., Karimabadi, H., and Acuna, M.: Hybrid simulation of solar wind interaction with magnetized asteroids: General characteristics, J. Geophys. Res., 107, D1487, doi:10.1029/2002JA009441, 2002.

Omidi, N., Blanco-Cano, X., Russel, C. T., and Karimabadi, H.: Dipolar magnetospheres and their characterization as a function of magnetic moment, Adv. Space Res., 33, 1996-2003, 2004.
Paty, C. and Winglee, R.: Multi-fluid simulations of Ganymede's magnetosphere, Geophys. Res. Lett., 31, L24806, doi:10.1029/2004GL021220, 2004.

Raeder, J., McPherron, R. L., Frank, L. A., Kokubun, S., Lu, G., Mukai, T., Paterson, W. R., Sigwarth, J. B., Singer, J. H., and Slavin, J. A.: Global simulation of the Geospace Environment Modeling substorm challenge event, J. Geophys. Res., 103, 381395, 2001.

Simon, S., Bagdonat, T., Motschmann, U., and Glassmeier, K.-H.: Plasma environment of magnetized asteroids: a 3-D hybrid simulation study, Ann. Geophys., 24, 407-414, 2006, http://www.ann-geophys.net/24/407/2006/.

Slinker, S. P., Fedder, J. A., Ruohoniemi, J. M., and Lyon, J. G.: Global MHD simulation of the magnetosphere for November 24, 1996, J. Geophys. Res., 106(A1), 361-380, 2001.

Spicer, D. S., Zalesak, Z. T., Löhner, R., and Curtis, S.: SIMULATION OF THE MAGNETOSPHERE WITH A NEW THREE DIMENSIONAL MHD CODE AND ADAPTIVE MESH REFINEMENT: PRELIMINARY RESULTS, Adv. Space Res., 18, 253-262, 1996.

Stasiewicz, K.: Nonlinear Alfvén, magnetosonic, sound, and electron inertial waves in fluid formalism, J. Geophys. Res., 110, A03220, doi:10.1029/2004JA010852, 2005.

Stasiewicz, K.: Heating of the Solar Corona by Dissipative Alfvn solitons, Phys. Rev. Lett., 96, 175003, doi:10.1103/PhysRevLet.96.175003, 2006.

Sydora, R. D., Sauer, K., and Silin, I.: Coherent whistler waves and oscillitons formation: Kinetic simulations, Geophys. Res. Lett., 34, L22105, doi:10.1029/2007GL031839, 2007.

Trávnicek, P., Hellinger, P., and Schriver, D.: Structure of Mercury's magnetosphere for different pressure of the solar wind: Three dimensonal hybrid simulations, Geophys. Res. Lett., 34, L05104, doi:10.1029/2006GL028518, 2007.

Walker, R. J., Ogino, T. T., Raeder, J., and Maha Ashour-Abdalla: A Global Magnetohydrodynamic Simulation of the Magnetosphere When the Interplanetary Magnetic Field is Southward: The Onset of Magnetotail Reconnection, J. Geophys. Res., 98, 1723517249, 1993.

Wang, Z. and Kivelson, M.: Asteroid interaction with solar wind, J. Geophys. Res., 101, 24479-24493, 1996.

Whang, Y. C.: Magnetospheric magnetic field of Mercury, J. Geophys. Res., 82(7), 1024-1030, 1977. 